TITLE:

\title{
Susceptibility cancellation of a microcoil wound with a paramagnetic-liquid-filled copper capillary.
}

$\operatorname{AUTHOR}(\mathrm{S})$ :

Takeda, Kazuyuki; Takasaki, Tomoya; Takegoshi, K

\section{CITATION:}

Takeda, Kazuyuki ...[et al]. Susceptibility cancellation of a microcoil wound with a paramagnetic-liquid-filled copper capillary.. Journal of magnetic resonance 2015, 258: 1-5

ISSUE DATE:

2015-09

URL:

http://hdl.handle.net/2433/201600

RIGHT:

(C) 2015. This manuscript version is made available under the CC-BY-NC-ND 4.0 license

http://creativecommons.org/licenses/by-nc-nd/4.0/; The full-text file will be made open to the public on 30 September 2017 in accordance with publisher's 'Terms and Conditions for Self-Archiving'.; この論文は出版社版でありません。引 用の際には出版社版をご確認じ利用ください。; This is not the published version. Please cite only the published version. 


\title{
Susceptibility cancellation of a microcoil
}

\section{wound with a paramagnetic-liquid-filled}

\section{copper capillary}

\author{
Kazuyuki Takeda*, Tomoya Takasaki, K. Takegoshi \\ Division of Chemistry, Graduate School of Science, Kyoto University, 606-8502 \\ Kyoto, Japan
}

\begin{abstract}
Even though microcoils improve the sensitivity of NMR measurement of tiny samples, magnetic-filed inhomogeneity due to the bulk susceptibility effect of the coil material can cause serious resonance-line broadening. Here, we propose to fabricate the microcoil using a thin, hollow copper capillary instead of a wire and fill paramagnetic liquid inside the capillary, so as to cancel the diamagnetic contribution of the copper. Susceptibility cancellation is demonstrated using aqueous solution of $\mathrm{NiSO}_{4}$. In addition, the paramagnetic liquid serves as coolant when it is circulated through the copper capillary, effectively transferring the heat generated by radiofrequency pulses.
\end{abstract}

Key words: microcoil; bulk magnetic susceptibility; copper capillary; paramagnetic liquid; susceptibility cancellation

\footnotetext{
* corresponding author

Email address: takezo@kuchem.kyoto-u.ac.jp (Kazuyuki Takeda).
} 
For NMR measurements of mass-limited samples, a microcoil is advantageous in terms of the sensitivity[1,2], while the price to pay is the reduced spectral resolution due to magnetic-field inhomogeneity caused by the bulk susceptibility effect (BMS) of the coil wire[2]. In general, a body with non-zero magnetic susceptibility is magnetized in the presence of an external magnetic field. As a consequence, an additional perturbative field arises in the vicinity of the body. Since the strength of the induced field decreases rapidly with the distance, the BMS effect is relatively small for NMR measurements using sample coils with the conventional size. However, in the case of the microcoils, the sample is inevitably close to the coil wire. Thus, the BMS effect can cause significant line broadening.

Copper, which is used for the coil material in many cases, is diamagnetic, so that a magnetization antiparallel to the external field develops. The profile of the induced magnetic field is determined by the geometry of the coil. In the case of a solenoid coil, the resultant field distribution contains high-order terms that are difficult to remove by the standard shimming technique.

So far, several schemes to suppress the BMS-induced field inhomogeneity have been proposed[3]. They include susceptibility matching[2,4,5], zero-susceptibility wire[6-8], and magic-angle coil spinning (MACS)[9-11]. It is also worth noting that the effect of susceptibility broadening is small in stripline probes[12,13]. Here, we report an alternative approach. Our idea is to make a microcoil with a thin capillary made of copper and to fill paramagnetic liquid solution inside the capillary. We aim at cancelling the diamagnetic contribution of the copper.

Our scheme, which is similar to that of zero-susceptibility wire, has a noteworthy feature that the net susceptibility can be modified simply by changing 
the concentration of the paramagnetic solute. This contrasts with the case of the zero-susceptibility wire, where such fine adjustment is formidable. Indeed, when Hoult and Ginsberg used a commercial zero-susceptibility wire in their studies on nuclear spin noise, they had to do an elaborate job of electroplating an additional copper layer, as they found that the diameter of their copperclad aluminum-core wire differed significantly from the nominal value[14]. In addition, the paramagnetic liquid can flow through the capillary, serving as effective coolant, as demonstrated below.

Thin copper capillaries are used for the electrodes of discharge machining devices and are commercially available. In this work, we used one with an outer diameter of $0.2 \mathrm{~mm}$ and an inner diameter of $0.1 \mathrm{~mm}$, which was purchased from Nippon Tokushukan MFG. CO., LTD. Since the copper capillary was fragile, we wound it gently into microcoils so as not to clog the passage. An example of copper-capillary-wound microcoils is shown in Fig. 1(a). Its inner diameter is $0.4 \mathrm{~mm}$, and the number of turns is 8 . Using a chip ceramic capacitor for balancing and a pair of surface mount trimmer capacitors (TZC3, Murata Electronics) for impedance matching, we fabricated a tank circuit resonating at ca. $300 \mathrm{MHz}$ on a printed circuit board (Fig. 1(b)). The Q factor of the circuit was measured to be 23. Each end of the coil penetrates the board through a conducting via, at which the electrical connection is established by soldering. To supply liquid, poly ether ether ketone (PEEK) tubes were connected at both ends of the copper capillary (Fig. 1(c)).

For paramagnetic liquid, we used aqueous solution of $\mathrm{NiSO}_{4}$. For molar concentration $\alpha$, the susceptibility at room temperature is given by $50.3 \alpha \times 10^{-6}$ in SI unit, while the susceptibility of our microcoil was measured to be $-10.6 \times 10^{-6}$ (vide infra). Since the volume fraction of the hollow region of the capillary is 
25 percent, we naively estimated the concentration $\alpha$ that annuls the net susceptibility to be $0.63 \mathrm{M}$.

The standard electrode potentials for nickel and copper are $-0.23 \mathrm{~V}$ and $+0.35 \mathrm{~V}$, respectively. Since the ionization tendency increases with the negative potential, $\mathrm{Ni}+\mathrm{Cu}^{2+} \rightarrow \mathrm{Ni}^{2+}+\mathrm{Cu}$, rather than $\mathrm{Ni}^{2+}+\mathrm{Cu} \rightarrow \mathrm{Ni}+\mathrm{Cu}^{2+}$, is favoured thermodynamically. Thus, dissolution of copper into the $\mathrm{NiSO}_{4}$ solution is thermodynamically unfavourable.

${ }^{1} \mathrm{H}$ NMR measurements were performed using a $7 \mathrm{~T}$ superconducting magnet at room temperature. The carrier frequency was $299.52 \mathrm{MHz}$. First, we carried out experiments with the microcoil without filling liquid inside the capillary. Fig. 2(a) shows a ${ }^{1} \mathrm{H}$ spectrum of $0.1 \mathrm{M}$ aqueous solution of $\mathrm{CuSO}_{4}$ in a quartz tube with an inner/outer diameter of $0.2 / 0.4 \mathrm{~mm}$. We found considerable broadening and distortion in the spectrum acquired with the microcoil, which were caused by the BMS effect of the microcoil. The full width at half maximum was $93 \mathrm{~Hz}$. For comparison, we also acquired a ${ }^{1} \mathrm{H}$ NMR spectrum of the same sample using a microcoil of the same geometry but wound with a solid copper wire with a diameter of $0.2 \mathrm{~mm}$ (Fig. 2(b)). We found that the sensitivity was comparable for both coils, while the broadening and distortion of the resonance line were less appreciable for the capillary-wound microcoil. In addition, we measured ${ }^{1} \mathrm{H}$ NMR of the same sample using a larger coil with a diameter of $5 \mathrm{~mm}$ (Fig. 2(c)). Here, the origin of the line width (38 Hz) was ascribed to the field inhomogeneity of our $7 \mathrm{~T}$ magnet used in the present study.

Next, we performed experiments by supplying aqueous solution of $\mathrm{NiSO}_{4}$ inside the copper capillary. No background ${ }^{1} \mathrm{H}$ signal due to the paramagnetic 
liquid was observed. As demonstrated in Fig. 3(a), the line width of the ${ }^{1} \mathrm{H}$ spectrum decreased with the concentration of $\mathrm{NiSO}_{4}$, reached the minimum $(52 \mathrm{~Hz})$ at $0.54 \mathrm{M}$. With increasing the $\mathrm{NiSO}_{4}$ concentration further, the spectral resolution degraded, because the paramagnetic contribution became too strong. As shown in Fig. 3(b), with the optimal concentration, the peak height was enhanced by $58 \%$ compared to that in the case of pure water (i.e., no $\mathrm{NiSO}_{4}$ dissolved). In addition, with increasing the overall paramagnetic contribution, the resonance line shifted to higher frequencies (Fig. 3(c)).

The optimal $\mathrm{NiSO}_{4}$ concentration of $0.54 \mathrm{M}$ was ca. $14 \%$ smaller than the estimated value of $0.63 \mathrm{M}$. In order to discuss the difference, we performed numerical analysis as follows. The longitudinal component $b_{z}\left(\mathbf{r} ; \mathbf{r}^{\prime}\right)$ of the magnetic field produced at point $\mathbf{r}=(x, y, z)$ by an infinitesimal magnetization $\mathbf{m}\left(\mathbf{r}^{\prime}\right) d \mathbf{r}^{\prime}$ at point $\mathbf{r}^{\prime}=\left(x^{\prime}, y,{ }^{\prime} z^{\prime}\right)$ is given by

$$
b_{\mathrm{z}}\left(\mathbf{r} ; \mathbf{r}^{\prime}\right)=-\frac{\mu_{0}}{4 \pi} \frac{\mathbf{m}\left(\mathbf{r}^{\prime}\right) d \mathbf{r}^{\prime}}{\left|\mathbf{r}-\mathbf{r}^{\prime}\right|^{3}}\left[1-\frac{3\left(z-z^{\prime}\right)^{2}}{\left|\mathbf{r}-\mathbf{r}^{\prime}\right|^{2}}\right]
$$

In our case, the magnetization density $\mathbf{m}\left(\mathbf{r}^{\prime}\right)$ is represented using the materialdependent magnetic susceptibility $\chi\left(\mathbf{r}^{\prime}\right)$ and the external-field strength $B_{0}$ as

$$
\mathbf{m}\left(\mathbf{r}^{\prime}\right)=\chi\left(\mathbf{r}^{\prime}\right) B_{0}
$$

The overall BMS-induced field $B_{\mathrm{m}}(\mathbf{r})$ at position $\mathbf{r}$ is obtained by performing integration

$$
B_{\mathrm{z}}(\mathbf{r})=\int d \mathbf{r}^{\prime} b_{\mathrm{z}}\left(\mathbf{r} ; \mathbf{r}^{\prime}\right)
$$

over the volume of the coil material and the paramagnetic liquid inside the copper capillary.

To estimate BMS-induced broadening, we carried out numerical calculations according to Eq. (3) for the geometry of the setup used in the experiments 
presented above. The actual susceptibility of the microcoil was determined with a SQUID fluxmeter (MPMS-XL7ACMKO, Quantum Design, Inc.) at $300 \mathrm{~K}$ to be $-10.6 \times 10^{-6}$.

Fig. 4(a) shows a contour plot of magnetic-field distribution in the plane that bisects the microcoil, which was calculated without taking paramagnetic liquid into account. That is, this calculation corresponds to the case of the hollow capillary shown in Fig. 2(a). Here, we assumed that the external magnetic field was perfectly homogeneous, so that the displayed inhomogeneity arises purely from the BMS effect. Then, we carried out calculations of the field distribution for various $\mathrm{NiSO}_{4}$ concentrations and found that $0.66 \mathrm{M}$ gave the minimum BMS-induced inhomogeneity, where far fewer contour lines appear (Fig. 2(b)).

Figs. 4(c) and 4(d) show histograms representing the field distribution for the hollow copper capillary and for the optimal $\mathrm{NiSO}_{4}$ concentration, which were obtained by evaluating $B_{\mathrm{z}}$ over the entire sample volume (a cylindrical region with a diameter of $0.2 \mathrm{~mm}$ and a length of $10 \mathrm{~mm}$ ). To incorporate the original field inhomogeneity of the $7 \mathrm{~T}$ magnet caused by reasons other than the BMS effect of the microcoil, we applied convolution operation to the histogram using a Gaussian function with the width of $38 \mathrm{~Hz}$. The resultant spectra, shown in Figs. 4(e) and 4(f), correspond to the experimental results shown in Fig. 2(a) without paramagnetic liquid and with the concentrationoptimized $\mathrm{NiSO}_{4}$ aqueous solution; the increase in the peak height was found to be consistent with the experimental observation.

The optimal $\mathrm{NiSO}_{4}$ concentration of $0.66 \mathrm{M}$ obtained from the numerical calculation was slightly higher than that of the aforementioned estimation $(0.63 \mathrm{M})$ 
from the volume fraction. This is reasonable, considering that the diamagnetic part is located in the outer layer of the coil material, i.e., slightly closer to the sample region than the paramagnetic core. Since the induced field decreases with the cube of the distance from the source, the optimal paramagnetic concentration should be higher than the concentration that would exactly cancel the net susceptibility.

However, the experimentally obtained optimal $\mathrm{NiSO}_{4}$ concentration of $0.54 \mathrm{M}$ was lower than the estimated value. Moreover, even with the experimentally optimized condition, the ${ }^{1} \mathrm{H}$ resonance line width $(52 \mathrm{~Hz})$ was larger than that $(38 \mathrm{~Hz})$ expected from the field inhomogeneity of the magnet used in the present study. The discrepancies may be explained by deformation of the copper material upon winding the microcoil.

Another interesting feature of the capillary-wound coil that we found in this work is that the temperature of the coil can be regulated by circulating the liquid. Microcoil NMR allows for irradiation of such strong radiofrequency pulses that was not feasible with the conventional coil[15-17], opening many potential applications in solid-state NMR where coherent manipulation of relatively strong spin interactions is of interest. So far, strong irradiation with the microcoil has found interest in the context of ${ }^{1} \mathrm{H}$ decoupling $[16,18,19]$ and multiple-quantum magic angle spinning (MQMAS)[20]. One problem regarding to the strong irradiation is coil heating. Since the radiofrequency power is eventually dissipated as heat in a small region around the microcoil, the strong irradiation can burn the microcoil, unless the coil is effectively cooled down.

To demonstrate the efficiency of cooling, we applied radiofrequency irradia- 
tion every second with a duration of $100 \mathrm{~ms}$ and a power of $19 \mathrm{~W}$, which corresponds to a nutation frequency of $500 \mathrm{kHz}$. We used an infrared camera (FLIR-i7) to monitor the temperature of the coil as well as of the probe circuit. Figs. 5(a)-(c) show heating observed without liquid flowing inside the coil capillary. The temperature indicated in the figure is that where the microcoil was located. The coil temperature increased up to 73 degrees, as we continued measurement for $500 \mathrm{~s}$, when we stopped the experiment to prevent the coil from burning.

Then, we applied the same sequence with circulating water (20 degrees) at a flow rate of $0.8 \mathrm{~mL} / \mathrm{min}$. As demonstrated in Figs. $5(\mathrm{~d})-(\mathrm{f})$, the coil temperature was found to increase only slightly. Interestingly, the thermography images revealed that the coolant carried away the heat of not only the microcoil but also of the entire resonant circuit including the capacitors and the circuit board. The water flow provides a reliable way of cooling the circuit compared to air blowing, as the former takes heat away from the whole region of the microcoil, while the latter does only on the side at which the air is blown.

To summarize, we proposed to use a thin copper capillary for the microcoil and fill the capillary with paramagnetic liquid. By adjusting the concentration of the paramagnetic solute, the overall bulk susceptibility effect can be suppressed significantly, leading to the improved spectral resolution. The paramagnetic liquid also serves for coolant. By circulating the liquid through the capillary-wound microcil, significant suppression of coil heating is realized. We plan to assemble this into the microcoil MAS system. 


\section{References}

[1] T. L. Peck, R. L. Magin, P. C. Lauterbur, Design and Analysis of Microcoils for NMR Microscopy, Journal of Magnetic Resonance, B108 (1995) 114-124.

[2] D. L. Olson, T. L. Peck, A. G. Webb, R. L. Magin, J. V. Sweedler, HighResolution Microcoil ${ }^{1} \mathrm{H}-\mathrm{NMR}$ for Mass-Limited, Nanoliter-Volume Samples, Science, 270 (1995) 1967-1970.

[3] M. E. Lacey, R. Subramanian, D. L. Olson, A. G. Webb, J. V. Sweedler, HighResolution NMR Spectroscopy of Sample Volumes from $1 \mathrm{~nL}$ to $10 \mu \mathrm{L}$, Chemical Reviews, 99 (1999) 3133-3152.

[4] B. Behnia, A. G. Webb, Limited-sample NMR using solenoidal microcoils, perfluorocarbon plugs, and capillary spinning, Analytical Chemistry, 70 (1998) $5326-5331$.

[5] R. Kc, Y. N. Gowda, D. Djukovic, I. D. Henry, G. H. J. Park, D. Raftery, Susceptibility-matched plugs for microcoil NMR probes, Journal of Magnetic Resonance, 205 (2010) 63-68.

[6] R. Kc, I. D. Henry, G. H. J. Park, A. Aghdasi, D. Raftery, New solenoidai microcoil NMR probe using zero-susceptibility wire, Concepts in Magnetic Resonance Part B, 37 (2010), 13-19.

[7] F. O. Zelaya, S. Crozier, S. Dodd, R. Mckenna, D. M. Doddrell, Measurement and Compensation of Field Inhomogeneities Caused by Differences in Magnetic Susceptibility, Journal of Magnetic Resonance, A115 (1995) 131-136.

[8] N. Soffe, J. Boyd, M. Leonard, The Construction of a High-Resolution $750 \mathrm{MHz}$ Probehead. Journal of Magnetic Resonance, A116 (1995) 117-121.

[9] D. Sakellariou, G. Le Goff, J.-F. Jacquinot, High-resolution, high-sensitivity 
NMR of nanolitre anisotropic samples by coil spinning, Nature, 447 (2007), $694-697$.

[10] M. Inukai, K. Takeda, Double-resonance magic angle coil spinning, Journal of Magnetic Resonance, 202 (2010) 274-278.

[11] A. Wong, B. Jimnez, X. Li, E. Holmes, J. K. Nicholson, J. C. Lindon, D. Sakellariou, Evaluation of High Resolution Magic-Angle Coil Spinning NMR Spectroscopy for Metabolic Profiling of Nanoliter Tissue Biopsies, Analytical Chemistry, 84 (2012) 3843-3848.

[12] Y. Maguire, I. L. Chuang, S. Zhang, N. Gershenfeld, Ultra-small-sample molecular structure detection using microslot waveguide nuclear spin resonance, Proceedings of the National Academy of Sciences, 104 (2007) 9198-9203.

[13] P. J. M. Van Bentum, J. W. G. Janssen, A. P. M. Kentgens, J. Bart, J. G. E. Gardeniers, Stripline probes for nuclear magnetic resonance, Journal of Magnetic Resonance, 189 (2007) 104-113.

[14] D. I. Hoult, N. S. Ginsberg, The quantum origins of the free induction decay signal and spin noise, Journal of Magnetic Resonance, 148 (2001) 182-199.

[15] K. Yamauchi, J. W. G. Janssen, A. P. M. Kentgens, Implementing solenoid microcoils for wide-line solid-state NMR. Journal of Magnetic Resonance, 167 (2004) 87-96.

[16] H. Janssen, A. Brinkmann, E. R. H. van Eck, P. J. M. van Bentum, A. P. M. Kentgens, Microcoil high-resolution magic angle spinning NMR spectroscopy. Journal of the American Chemical Society, 128 (2006), 8722-8723.

[17] A. P. M. Kentgens, J. Bart, P. J. M. van Bentum, A. Brinkmann, E. R. H. van Eck, J. G. E. Gardeniers, J. W. G. Janssen, P. Knijn, S. Vasa, M. H. W. Verkuijlen, High-resolution liquid- and solid-state nuclear magnetic resonance 
of nanoliter sample volumes using microcoil detectors. Journal of Chemical Physics, 128 (2008) 052202.

[18] S. K. Vasa, H. Janssen, E. R. H. van Eck, A. P. M. Kentgens, High-resolution solid-state ${ }^{13} \mathrm{C} \mu \mathrm{MAS}$ NMR with long coherence life times. Physical Chemistry Chemical Physics, 13 (2011), 104-106.

[19] K. Takeda, Microcoils and microsamples in solid-state NMR. Solid State Nuclear Magnetic Resonancee, 47-48 (2012) 1-9.

[20] M. Inukai, K. Takeda, Studies on Multiple-Quantum Magic-Angle- Spinning NMR of Half-Integer Quadrupolar Nuclei Under Strong rf Pulses with a Microcoil. Concepts in Magnetic Resonance, B33 (2008) 115-123. 
(a)
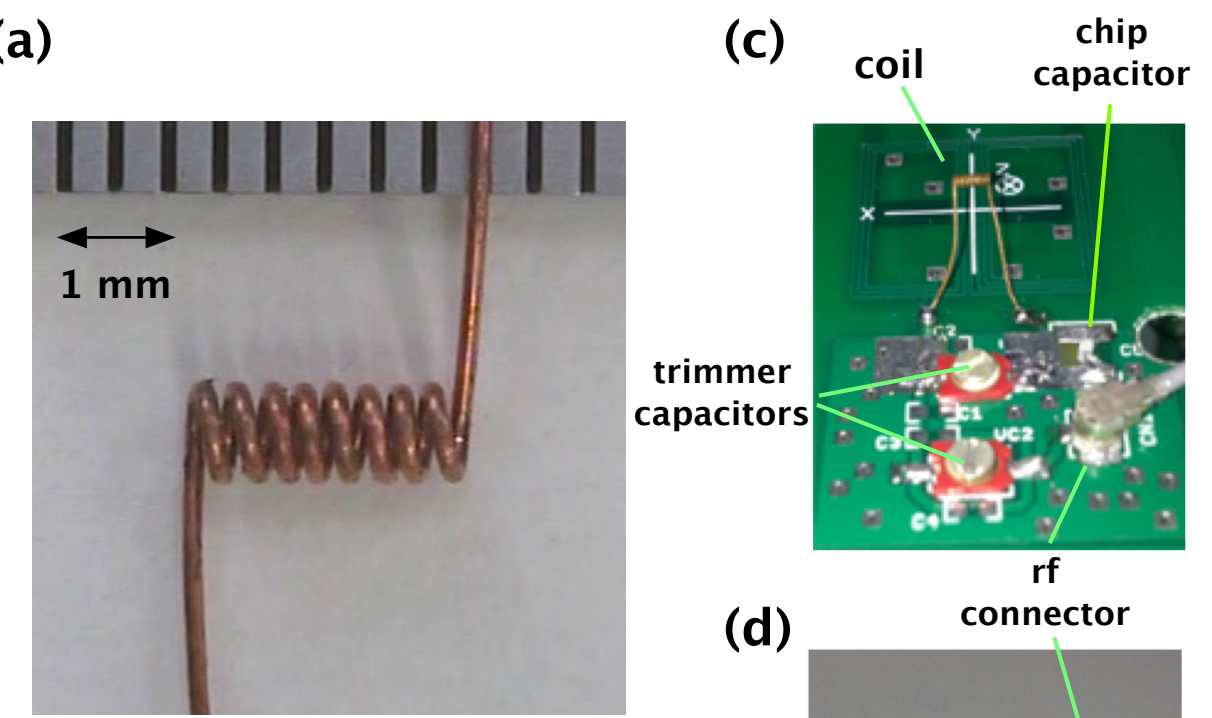

(d)

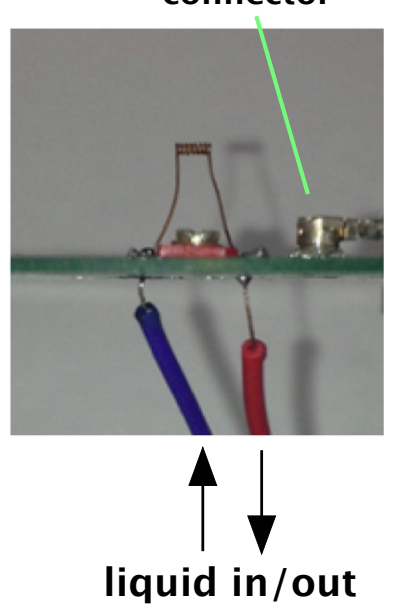

Fig. 1. (a) A photo of a microcoil wound with a copper capillary with an inner diameter of $0.1 \mathrm{~mm}$ and an outer diameter of $0.2 \mathrm{~mm}$. (b) Schematic drawing of the cross section of the capillary. (c) A probehead composed of the microcoil, a chip capacitor, and trimmer capacitors. (d) A side view of the probe. Tubes are connected to both end of the copper capillary to supply liquid. 

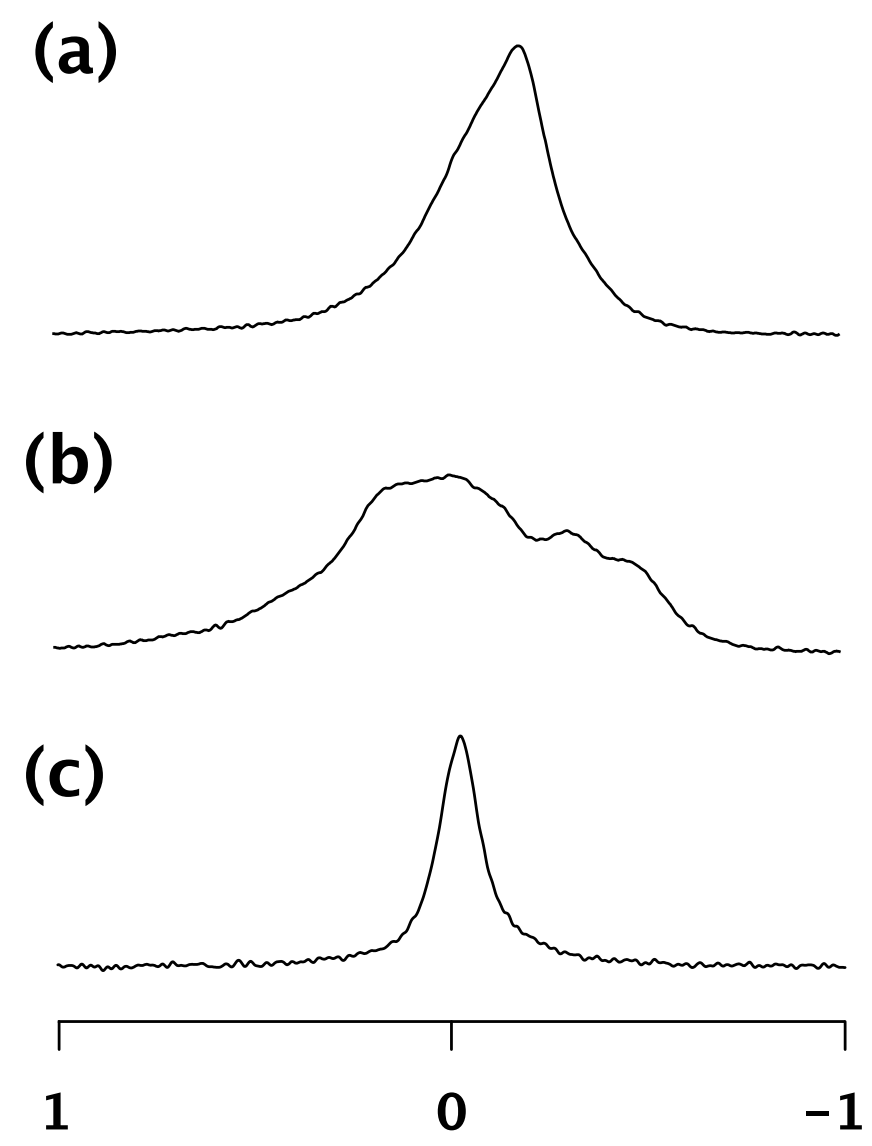

Chemical shift [ppm]

Fig. 2. ${ }^{1} \mathrm{H}$ spectra of aqueous solution of $0.1 \mathrm{M} \mathrm{CuSO}_{4}$ in a quartz tube with an inner diameter of $0.2 \mathrm{~mm}$ and a length of $10 \mathrm{~mm}$. The resonance frequency was 299.52 MHz. (a) was acquired using the microcoil probe shown in Fig. 1 without filling paramagnetic liquid, while (b) was obtained with a microcoil of the same geometry but wound with a $0.2 \mathrm{~mm}$ solid copper wire. (c) shows a ${ }^{1} \mathrm{H}$ spectrum of the same sample measured with a larger coil with a diamter of $5 \mathrm{~mm}$. 
(a)

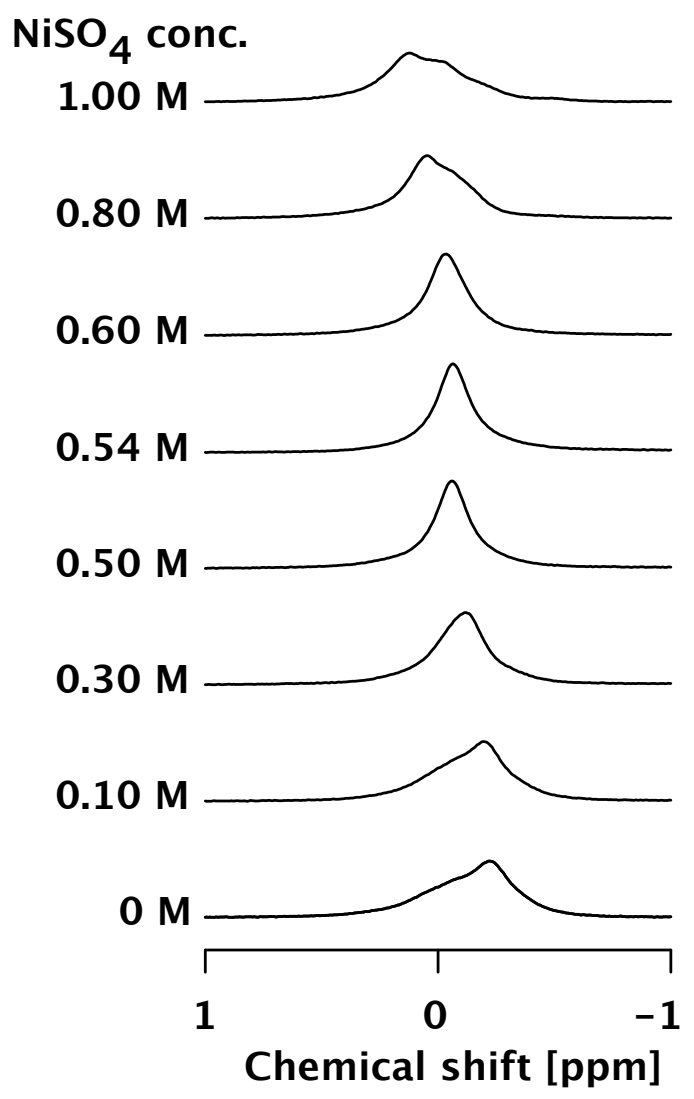

(b)

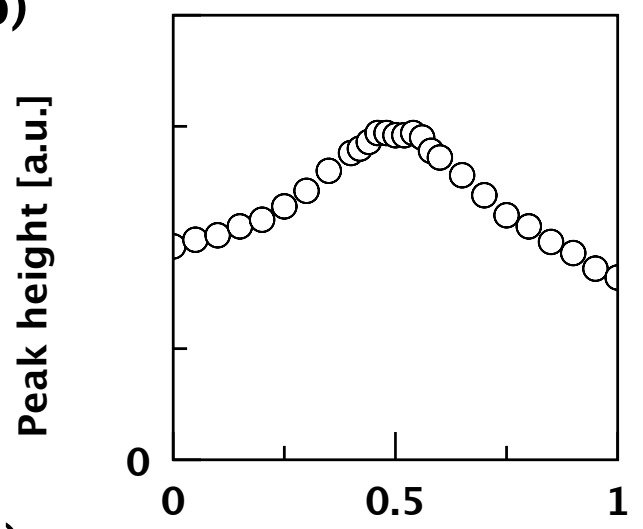

(c)

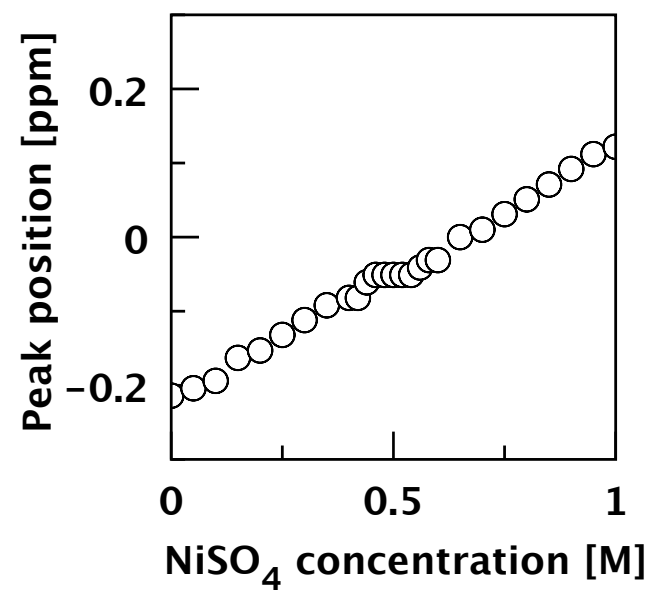

Fig. $3 .{ }^{1} \mathrm{H}$ spectra of aqueous solution of $0.1 \mathrm{M} \mathrm{CuSO}_{4}$ obtained using a copper-capillary-wound microcoil filled with $\mathrm{NiSO}_{4}$ aqueous solution with varying concentration. (b) (c) $\mathrm{NiSO}_{4}$ concentration dependences of the peak height and the peak position. 
(a)

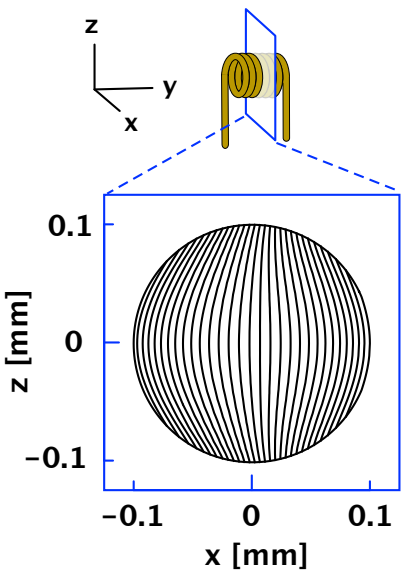

(c)

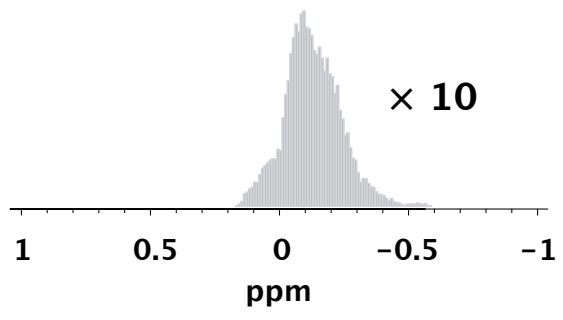

(e)

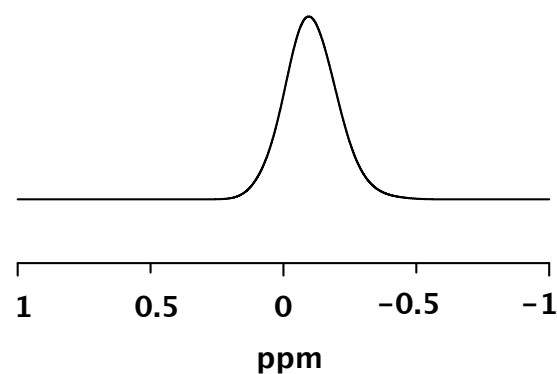

(b)

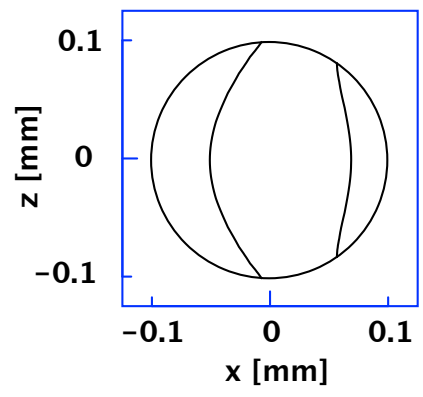

(d)

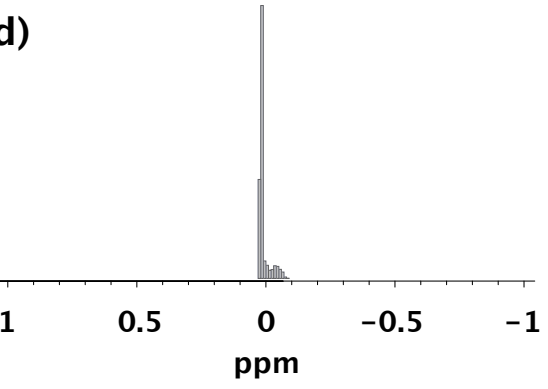

(f)

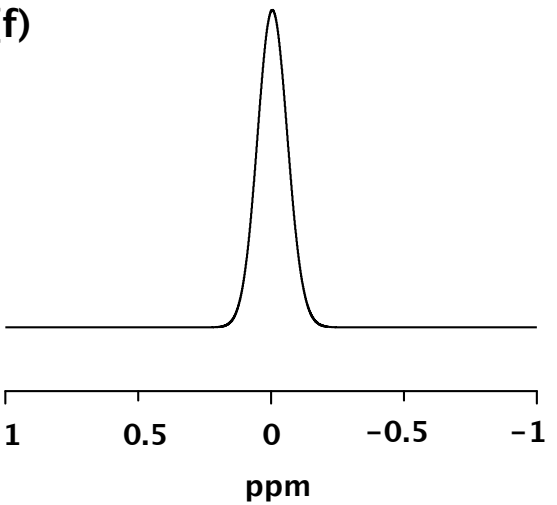

Fig. 4. (a) A contour plot of magnetic field distribution in a plane bisecting the microcoil calculated for the case where no paramagnetic liquid is supplied. (b) is a result obtained with $0.66 \mathrm{M}$ aqueous solution of $\mathrm{NiSO}_{4}$ filled inside the copper capillary. Contour lines are drawn every 0.005 ppm. (c)(d) Histograms representing the field distribution over a cylindrical region with a diameter of $0.2 \mathrm{~mm}$ and a length of $10 \mathrm{~mm}$, which corresponds to the sample volume. (e)(f) were obtained by performing convolution operation to the histograms of (c) and (d) using a Gaussian function with a width of $38 \mathrm{~Hz}$. 


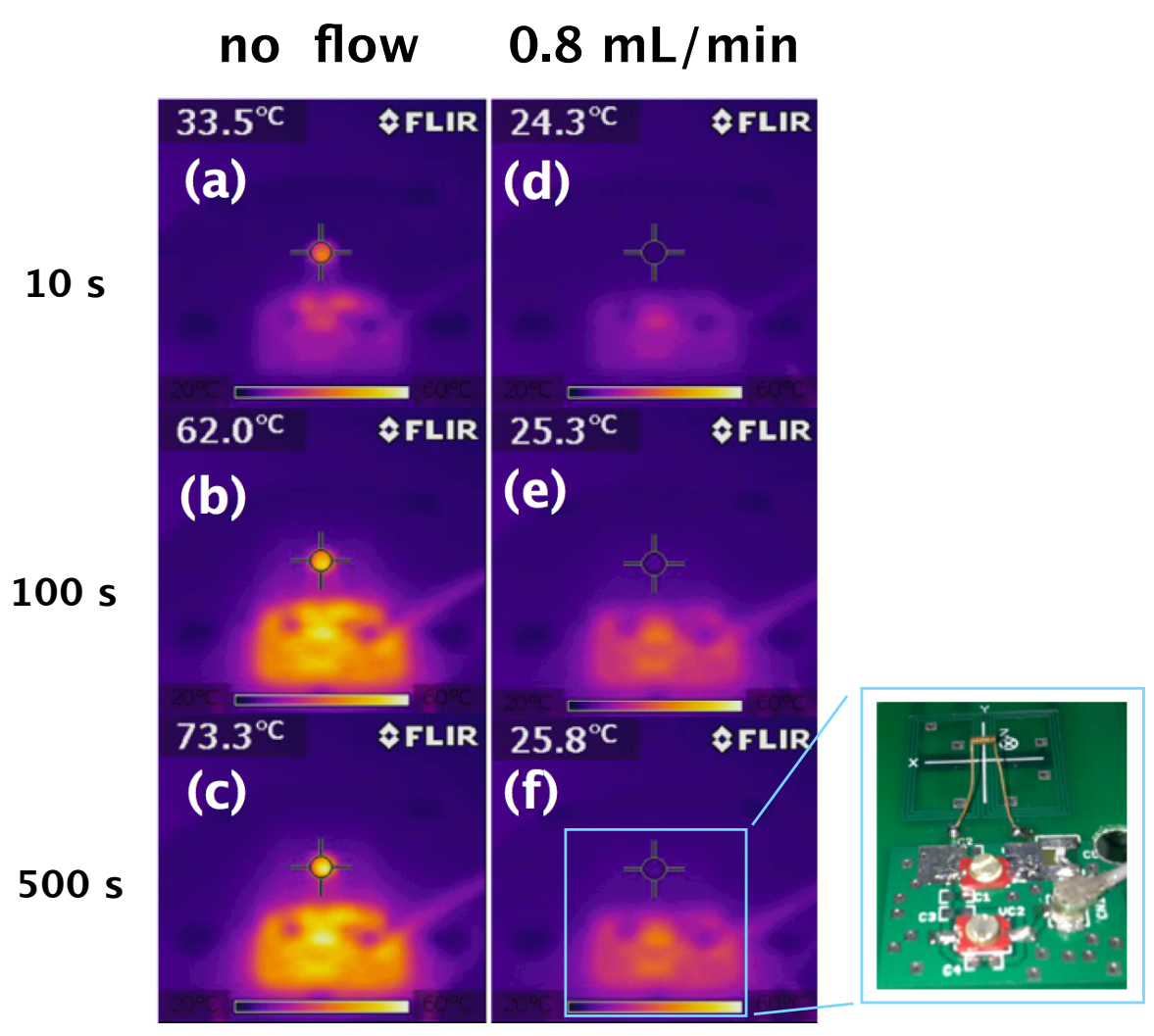

Fig. 5. Thermography images of the probe circuit under applications of radiofrequency pulses every one second at $300 \mathrm{MHz}$ with a power of $19 \mathrm{~W}$ and a duration of $100 \mathrm{~ms}$. In advance to this measurement, ${ }^{1} \mathrm{H}$ nutation experiments were performed and the radiofrequency power was found to correspond to a nutation frequency of $500 \mathrm{kHz} .(\mathrm{a})(\mathrm{b})(\mathrm{c})$ show images taken after $10 \mathrm{~s}, 100 \mathrm{~s}$, and $500 \mathrm{~s}$ after starting the experiment with no liquid flow inside the copper-capillary microcoil. (d)(e)(f) show results obtained by circulating 20 degree water at a flow rate of $0.8 \mathrm{~mL} / \mathrm{min}$. 